\title{
Trois prototypes de véhicules pour faire rouler les enseignements à l'ENSISA : un retour d'expérience
}

\author{
Rodolfo ORJUELA ${ }^{1,2,3}$ Jean-Charles PERUCHETTI ${ }^{1,2}$, Michel BASSET ${ }^{1,2,3}$ \\ rodolfo.orjuela@uha.fr \\ ${ }^{1}$ Université de Haute-Alsace (UHA) \\ ${ }^{2}$ Ecole Nationale Supérieure d'Ingénieurs Sud Alsace (ENSISA) \\ ${ }^{3}$ Laboratoire Modélisation, Intelligence, Processus, Systèmes (MIPS) EA233 \\ 12, rue des frères Lumière F-68093 MULHOUSE CEDEX
}

\begin{abstract}
RESUME : les projets tutorés et la participation aux clubs scientifiques constituent deux moyens intéressants de placer l'étudiant au cœur du processus de sa propre formation universitaire. Au sein des formations liées aux sciences de l'ingénieur, ces moyens sont une façon motivante pour le futur ingénieur de se confronter à une problématique réelle, de mettre en application les notions théoriques étudiées et d'acquérir des compétences autres que scientifiques (gestion d'un projet, travail en groupe, autonomie, etc.) nécessaires à son futur métier d'ingénieur. Nous présentons, dans cette communication, trois réalisations techniques issues de cette démarche pédagogique à l'ENSISA. Il s'agit de trois prototypes de véhicules, à savoir, un buggy électrique, une Peugeot 206 hybride et une voiture de course biplaces électrique.
\end{abstract}

Mots clés : plateformes expérimentales; véhicule électrique; véhicule hybride; enseignement actif.

\section{INTRODUCTION}

Un élément important dans toute formation universitaire en général et dans la formation universitaire liée aux sciences de l'ingénieur en particulier, est la mise en application des concepts théoriques présentés. Le futur ingénieur doit être en mesure d'apporter des solutions à des problèmes concrets à partir de connaissances théoriques et techniques, comme l'a très bien résumé Neil Armstrong dans cette phrase ${ }^{1}$ : Science is about what is. Engineering is about what can be.

Dans les approches d'enseignement classiques, cette confrontation à la réalité se fait dans un premier temps au cours des séances de Travaux Dirigés (TD) permettant à l'étudiant d'assimiler les concepts théoriques exposés en cours, puis par une application concrète à travers les séances de Travaux Pratiques (TP) dans un second temps. Au lieu de confronter l'élève à une situation réelle d'ingénieur, les TP visent plutôt une vérification de certains concepts théoriques par l'expérience. Par conséquent, les séances de TP ne permettent pas à l'étudiant de se confronter à une situation réelle (démarche d'ingénieur) où la solution à un problème n'est pas a priori connue. Cette forme d'enseignement présente donc certaines limites dans la mesure où l'étudiant n'est pas actif (proposition de solutions limitées, etc.) au cours du processus de formation.

Il peut être intéressant alors d'intégrer des compléments pédagogiques sous la forme, par exemple, de projets tutorés [1] permettant à l'étudiant d'exploiter

\footnotetext{
${ }^{1}$ Neil Armstrong at the 2000 National Press Club, which named him among those who completed the 20th century's Top 20 Engineering Achievements
}

les enseignements acquis d'une part et d'acquérir d'autres compétences transversales (autonomie, conduite de projet, etc.) extrêmement importantes pour sa future vie professionnelle et bien souvent délicates à obtenir lors d'une séance de TP d'autre part. Conscients de ce besoin, nous avons introduit progressivement depuis plusieurs années des projets tutorés à l'Ecole Nationale Supérieure d'Ingénieurs Sud Alsace (ENSISA) tout au long du cycle de formation d'ingénieur. Aussi, la création des clubs d'étudiants à vocation scientifique et technique au sein de l'école permet également aux élèves ingénieurs de s'investir dans un projet concret qui soit à la fois en dehors du cadre scolaire et motivé par une démarche complètement personnelle. L'objectif de ces projets (tutorés ou issus d'un club) est d'offrir la possibilité à l'étudiant de sortir du cadre académique traditionnel, s'appuyant sur la transmission de la connaissance, et d'aller vers un enseignement « constructiviste » [2] où il devient actif et situé au cœur du processus de formation.

Toutefois, trouver un support pédagogique sous la forme d'une plate-forme expérimentale visant la mise en place de ces projets reste une tâche délicate. Il est important de proposer des sujets qui constituent un défi raisonnable pour l'élève ingénieur, c.-à-d. assez difficiles pour créer un challenge mais dont les résultats escomptés restent atteignables afin d'éviter une situation d'échec. Les Robots LEGO ${ }^{\circledR}$ Mindstorms NXT (RNXT) sont mis à profit en tant que supports pédagogiques à l'ENSISA pour des projets en $1^{\text {ère }}$ année [3]. Ces robots à coût relativement faible, très robustes, offrent la possibilité de proposer un grand nombre de manipulations. Il reste cependant difficile de confronter l'étudiant à une vraie problématique industrielle à l'aide de ce support. Pour cette raison, d'autres plateformes expérimentales à caractère plus industriel sont 
utilisées à l'ENSISA à savoir des véhicules automobiles.

Nous avons acquis au fil des années trois véhicules automobiles : un buggy, une Peugeot 206 et une voiture de course biplace. Ces véhicules servent de support aussi bien pour des projets tutorés que pour des projets de l'ECARTEAM, l'un de clubs scientifiques de l'école. Les projets proposés s'articulent autour du problème posé par leur transformation en véhicule électrique ou hybride. Ce problème industriel d'actualité offre un éventail important de projets de complexité variée qui vont de la conception mécanique de la chaîne de propulsion électrique, jusqu'à l'établissement des lois de commande en passant par la modélisation, l'acquisition de signaux, l'électronique, électrotechnique, l'informatique, les réseaux, le traitement du signal, la programmation, etc. D’une façon générale, il est possible d'imaginer une grande variété de projets interdisciplinaires en relation avec les quatre filières formation initiale à l'ENSISA : automatique et systèmes, mécanique, informatique et réseaux et textile et fibres. Ces véhicules constituent également un vecteur liant les activités de recherche et d'enseignement. Travailler sur ces véhicules constitue donc une source importante de motivation pour les étudiants.

Cette communication a pour objectif de présenter trois réalisations techniques, un buggy électrique, une Peugeot 206 hybride et une voiture de course biplace électrique. Ces réalisations techniques sont le résultat des projets tutorés des étudiants de l'ENSISA et en particulier des membres du club ECARTEAM. Cette communication est structurée comme suit. La Section 2 expose succinctement l'organisation de projets tutorés et de clubs à l'ENSISA. La Section 3 présente le travail réalisé pour aboutir à un buggy électrique à partir d'une version thermique. Les résultats de la transformation d'une Peugeot 206 en un véhicule hybride sont illustrés dans la Section 4. Quant à la Section 5, elle propose quelques résultats issus de la conception de la chaîne de propulsion électrique d'un véhicule de course biplace. En guise de conclusion, un bilan pédagogique est proposé au cours de la Section 6.

\section{DEMARCHE PEDAGOGIQUE}

Afin de donner la possibilité à l'étudiant d'aller vers un enseignement dit «par projet »[1], deux moyens pédagogiques conduisant à une mise en situation réelle sont proposés à l'ENSISA :

1. les projets tutorés qui s'intègrent dans la maquette pédagogique de chaque filière,

2. les projets des clubs scientifiques issus d'une démarche personnelle de l'étudiant.

\subsection{Projets tutorés}

Des projets tutorés sont proposés tout au long de la formation d'ingénieur. Nous présentons ici le cadre des projets tutorés en $2^{\text {ème }}$ année. D'une durée de six semaines à temps plein, représentant environ $200 \mathrm{~h}$ de travail personnel, ces projets se tiennent à la fin de la $2^{\text {ème }}$ année. Un problème (fourni sous la forme d'un cahier des charges des performances attendues) relativement complexe est proposé à un binôme d'étudiants qui doit proposer une solution en quasi-autonomie. Les étudiants sont ainsi confrontés à la recherche des solutions techniques mais aussi aux problèmes liés à la gestion du projet (planning, communication, contraintes, etc.). La période de projet se finalise par un exercice de communication où chaque groupe, sur la base d'un poster qu'il a réalisé, présente son travail à l'ensemble des étudiants (de $1^{\text {ère }}$ et $2^{\text {ème }}$ année) et aux membres du corps enseignant.

\subsection{Les clubs scientifiques}

L'ENSISA favorise l'émergence de clubs à vocation scientifique ou technique, issus d'une démarche personnelle des étudiants. Un local ou une salle est mis à disposition du club suivant les besoins. Ces clubs sont ouverts à tous les étudiants quelle que soit leur filière. Les clubs sont présentés dès le début de la $1^{\text {ère }}$ année au cours de la semaine d'intégration. Le temps consacré au club se fait en dehors de l'emploi du temps consacré aux cours, TDs et TPs. La participation active à un club donne lieu à une note d'investissement personnel.

Nous présentons ici le club ECARTEAM ${ }^{2}$ en lien direct avec les véhicules présentés. La création de ce club en 2007 a été principalement motivée par un groupe d'élèves souhaitant représenter l'ENSISA au Trophée organisé par la Société des Ingénieurs de l'Automobile (SIA). L'objectif de ce trophée est de concevoir et de réaliser un véhicule hybride sur la base d'un véhicule grand public en respectant un cahier des charges très précis (règles de sécurité, capacités de freinage, performance, régularité, etc.) imposé par la SIA pour les compétitions de rallye. Toutefois, les activités du club ne se limitent pas à la participation à ce Trophée. En effet, le club dispose de trois prototypes de véhicules permettant de proposer d'autres projets pour toutes les filières de l'école. Ces véhicules sont exposés régulièrement dans différents évènements grand public : festival international de l'automobile, rallye de France (à Mulhouse), journées de portes ouvertes, etc.

\section{DU BUGGY THERMIQUE A ELECTRIQUE}

Ce buggy (voir Figure 1), prévu à l'origine pour être transformé en véhicule solaro-éolien, est utilisé principalement comme support pédagogique pour des projets tutorés de $2^{\text {ème }}$ et $3^{\text {eme }}$ année du cycle de formation. Il est facile à appréhender pour les étudiants du fait de sa conception épurée (conduite simple) et constitue un moyen ludique pour les motiver. D'une façon générale, les projets proposés portent sur la conception et la mise

\footnotetext{
2 http://www.ecarteam.fr/
} 
en œuvre des solutions afin de remplacer la motorisation thermique du buggy par une propulsion électrique.
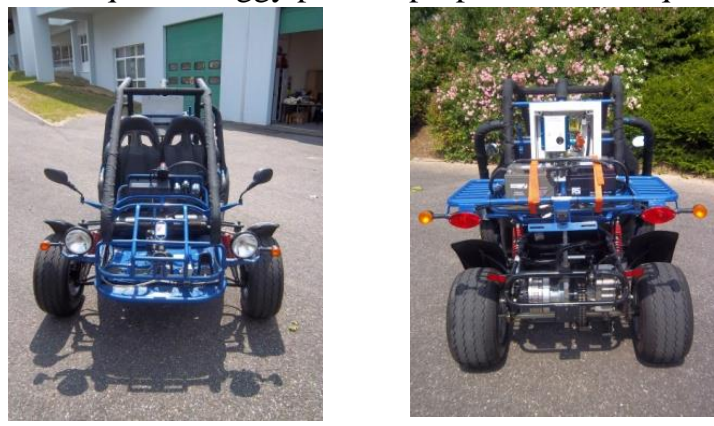

Figure 1 Buggy électrique

\subsection{Caractéristiques techniques originales}

Le véhicule utilisé comme base est un buggy modèle Raider MAX175D, 2 places, $175 \mathrm{~cm}^{3}, 2$ places fabriqué par la société DAZON ARIZONA. Ce buggy est homologué pour la route sans port de casque obligatoire. Le Tableau 1 présente quelques caractéristiques techniques du buggy d'origine.

\begin{tabular}{|l|l|}
\hline Motorisation & 4 temps, mono cylindre $175 \mathrm{cc}$ \\
\hline Boîte de vitesse & Automatique, centrifuge \\
\hline Vitesse max & $63 \mathrm{~km} / \mathrm{h}$ \\
\hline Poids & $235 / 255 \mathrm{~kg}$ \\
\hline Long./Larg./H. & $2300 / 1460 / 1420 \mathrm{~mm}$ \\
\hline Prix d'achat & 3000 euros \\
\hline \multicolumn{2}{|c|}{ Tableau 1. Caractéristiques techniques du Buggy }
\end{tabular}

\subsection{Chaîne de propulsion électrique}

La propulsion du buggy est assurée par un moteur LEM 170 LMC à courant continu, fonctionnant avec une tension de 12 à $48 \mathrm{~V}$, absorbant un courant nominal de $140 \mathrm{~A}$, fournissant une vitesse de rotation de $3264 \mathrm{tr} / \mathrm{min}$ et un couple de $16.3 \mathrm{Nm}$. Il est alimenté par 4 batteries $12 \mathrm{~V}-72 \mathrm{Ah}$. L'intégration du moteur électrique est illustrée sur la Figure 2.

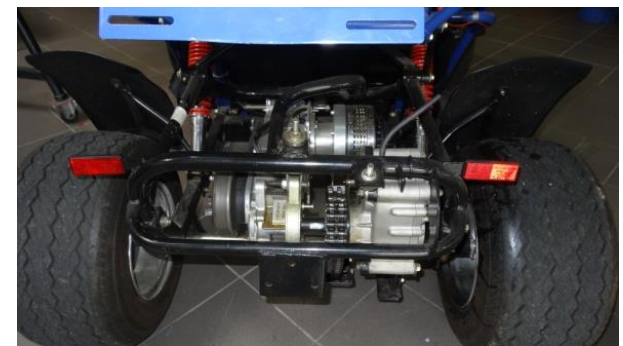

Figure 2 Mise en auvre de la motorisation du buggy

Compte tenu de sa taille et de sa puissance, ce moteur électrique présente un très bon compromis entre la puissance fournie et le poids avec un rendement supérieur à 90\%. Par ailleurs, il est capable de fournir un fort couple à faible vitesse, une caractéristique particulièrement intéressante pour les applications du véhicule électrique. Afin de réguler la vitesse du moteur électrique, un variateur AXE 4834 RI ALLTRAX pouvant fournir jusqu'à $200 \mathrm{~A}$ en sortie et alimenté en $48 \mathrm{~V}$, a été choisi dans un premier temps. Dans un futur proche, le buggy sera équipé d'un variateur Kelly bidirectionnel qui permettra la récupération d'énergie au freinage.

\subsection{Témoignage}

«Pour moi, le projet Buggy est un projet concret consistant à l'identification, la conception, la réalisation et la validation dans un temps imparti. Un vrai défi en termes de gestion de projet et de compétence pluridisciplinaire (..) les difficultés rencontrées sont la rédaction d'un cahier des charges réalisable (identifier) et certaines contraintes logistiques et administratives. » Florent CASASOLA filière Automatique et Systèmes. Diplômé en 2012. Actuellement doctorant au Laboratoire MIPS

\section{CONCEPTION D'UNE VOITURE HYBRIDE}

L'achat de ce véhicule (voir Figure 3) a été principalement motivé par le club ECARTEAM souhaitant représenter l'ENSISA au Trophée SIA. D'autres écoles d'ingénieurs ont également manifesté l'intérêt pour ce Trophée avec des réalisations très intéressantes [4]. A plusieurs reprises, l'ENSISA a participé avec la Peugeot 206 hybride au Trophée SIA. Ce véhicule est actuellement utilisé aussi bien pour les projets de l'ECARTEAM que pour des projets tutorés.
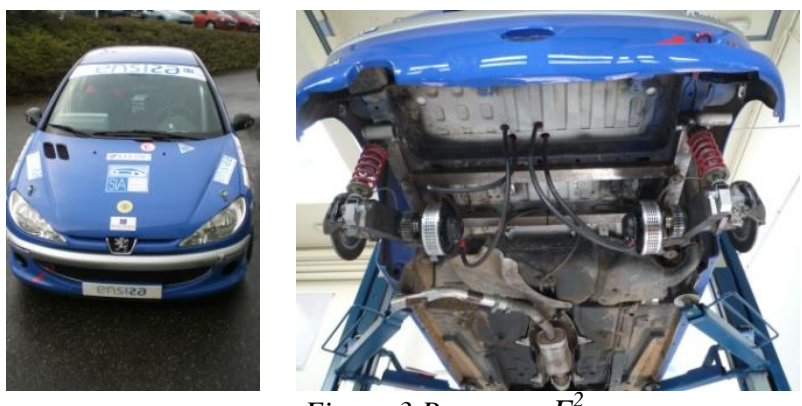

Figure 3 Prototype $E^{2}$

Le prototype réalisé, baptisé $\mathrm{E}^{2}$, est le résultat de la transformation d'une Peugeot 206 S16 initialement à motorisation thermique en un véhicule hybride comportant deux modes de motorisation, l'un thermique et l'autre électrique [5]. L'originalité de la solution proposée porte principalement sur l'utilisation de deux moteurs électriques placés à proximité des roues arrière du véhicule (voir Figure 3 à droite), s'approchant ainsi du concept moteur/roue (Active Wheel) de Michelin [6].

\subsection{Caractéristiques techniques}

Le véhicule de base pour ce projet est une Peugeot 206 S16 modèle 1999 (homologuée pour les Rencontres Peugeot Sport) à motorisation thermique, 2 roues motrices. Le Tableau 2 présente quelques caractéristiques techniques de la voiture d'origine.

\begin{tabular}{|l|l|}
\hline Motorisation & 4 cylindres, $1997 \mathrm{cc}$ \\
\hline Boîte de vitesse & Manuelle, 5 rapports \\
\hline
\end{tabular}




\begin{tabular}{|l|l|}
\hline Vitesse max & $211 \mathrm{~km} / \mathrm{h}$ \\
\hline Poids & $1064 \mathrm{~kg}$ \\
\hline Long./Lar./H. & $3830 / 1650 / 1430 \mathrm{~mm}$ \\
\hline Prix d'achat & 4000 euros \\
\hline
\end{tabular}

Tableau 2. Caractéristiques techniques de la 206

\subsection{Conception de la motorisation hybride}

L'idée mise en œuvre consiste à conserver le moteur thermique d'origine en traction sur les roues avant et d'ajouter deux moteurs électriques au niveau des roues arrière (voir Figure 3 à droite) afin de mettre en place une propulsion électrique. Un véhicule hybride à quatre roues motrices est ainsi obtenu. La Figure 4 illustre le schéma de principe de l'architecture proposée. Cette architecture d'hybridation est utilisée par exemple dans le concept-car Citroën C-Metisse présenté en 2006 ou plus récemment par le concept-car 5 by Peugeot Hybrid4 Concept présenté en 2010.

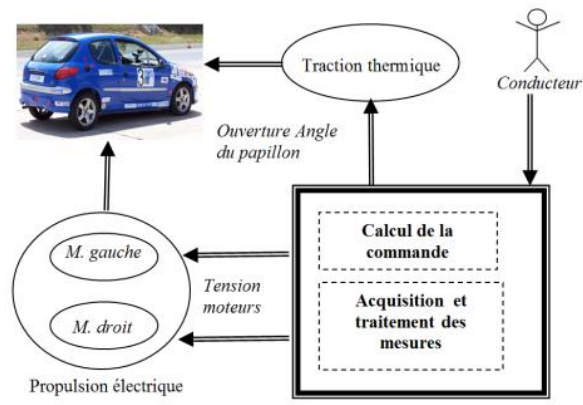

Figure 4 Schéma de principe pour la motorisation hybride

Pour le choix de la motorisation électrique, il a été décidé de retenir des modèles analogues à celui du buggy. Le choix des moteurs électriques s'est porté sur le moteur LEM 200 LMC à courant continu, fonctionnant avec une tension de $72 \mathrm{~V}$, absorbant un courant nominal de $200 \mathrm{~A}$, fournissant une vitesse de rotation de $3600 \mathrm{tr} / \mathrm{min}$ et un couple de 33.3 N.m. Les variateurs utilisés sont de type AXE7245 ALLTRAX et l'alimentation des moteurs électriques se fait à partir de deux séries de 6 batteries embarquées dans le coffre du véhicule. Les batteries sont de type gel/plomb (12 V et $38 \mathrm{Ah}$ ) et délivrent une énergie globale de $5472 \mathrm{~W}$ (72 $\mathrm{V}-76 \mathrm{Ah})$.

La transformation du véhicule d'origine en un véhicule hybride passe par une série de modifications, les plus remarquables étant le remplacement du train arrière d'origine afin de loger la nouvelle propulsion électrique, l'instrumentation de la voiture afin d'effectuer l'acquisition des mesures nécessaires à la commande des motorisations thermique et/ou électrique, l'introduction d'organes de commande pour la gestion des deux motorisations, la modification partielle du circuit hydraulique pour la gestion des freins ainsi que de la partie hydraulique pour la gestion de la direction assistée.
La conception des algorithmes de commande pour la gestion des deux motorisations est un problème très intéressant à traiter pour un automaticien. L'un des challenges ici est la conception d'un différentiel logiciel permettant de distribuer les vitesses de rotation des moteurs électriques lors d'un virage. La gestion des deux motorisations est réalisée à l'aide d'une cible temps réel DS1104 de dSpace. Tous les algorithmes de commande pour la gestion des deux modes de motorisation sont programmés en Matlab/Simulink. Des essais en conditions réelles montrent la validité de la solution adoptée $e^{3 .}$ Actuellement, une étude de faisabilité sur la récupération d'énergie au freinage par l'utilisation de super condensateurs est menée.

\subsection{Application TouchCar pour téléphone portable}

Un bus CAN (Controller Area Network) a été installé dans la voiture permettant de relier chaque bloc optique et d'en contrôler les feux indépendamment grâce à des modules CAN conçus par Didier BRESCH un enseignant de l'ENSISA.

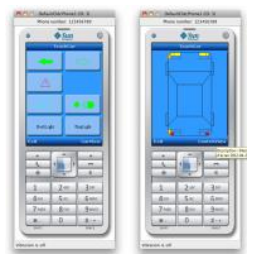

Figure 5 Application TouchCar pour téléphone portable

Une application (TouchCar) a été conçue de façon à contrôler les différents feux du véhicule grâce à un ordinateur et un écran tactile. L'application communique en réseau avec un serveur qui transmet les messages CAN aux modules. Réciproquement, chaque module transmet des messages CAN au serveur. Ces messages sont ensuite relayés aux applications connectées. L'application TouchCar utilise le bus CAN et permet de commander les feux mais aussi de visualiser un diagnostic des défauts du véhicule pour connaître les lampes défaillantes. Une variante de l'application a été programmée pour les téléphones portables et PDA afin de commander les feux de la voiture grâce à un téléphone portable. Les informations sur l'état du véhicule sont ainsi disponibles sur le téléphone et l'usager peut recevoir une alerte si une panne est détectée.

\subsection{Témoignages}

«Ce projet a été pour moi une source d'enrichissement autant dans le domaine de la théorie que celui de la pratique (...) mettre en pratique sur un vrai projet les connaissances acquisses tout au long de l'année (...) nous avoir appris à communiquer autour de celui-ci afin de trouver des solutions techniques et financières (...) le plus gros point bloquant surtout était d'ordre

${ }^{3}$ http://www.dailymotion.com/video/xig085 ecarteam-test-fontainefull-electric auto 
financier ou humain (difficulté à fédérer des étudiants) (...) pouvoir rester tard le soir à l'école avec plaisir quand le projet passionne (..) il m'a aussi ouvert les portes de l'industrie automobile car cette expérience a été une source de discussion et d'intérêt lors de mon entretien d'embauche à PSA.»

Jérôme FERRARI, filière Automatique et Systèmes. Diplômé en 2011. Co-fondateur de la société de robotique VOIDrobotic.

«Travailler sur un "vrai" projet qui n'a pas qu'un but éducatif, et donc rencontrer des problèmes qui ne sont pas les mêmes que ceux de l'année précédente et devoir les résoudre nous-même (défi personne) (...) travailler sur des problématiques proche de celle abordées dans l'industrie et aussi améliore son point de vue critique (...) le côté pluridisciplinaire, qui nous fait travailler sur les différents points techniques mais aussi la communication et l'organisation du projet. Ce projet permet surtout de s'amuser parce que les industries françaises sont beaucoup moins marrantes que les projets étudiant. »

Michaël JONDEAU, filière Automatique et Systèmes. Diplômé en 2011. Co-fondateur de la société de robotique VOIDrobotic.

«Le club a des projets intéressants liant mécanique/automatique/informatique/textile ce qui en fait un bon club pour mettre en avant les 4 filières de l'ENSISA. (...) J'ai pu développer des applications pour EcarTeam (projet TouchCar) (...) J'ai travaillé sur une série d'applications basées sur une architecture client/serveur pour interagir avec le bus CAN installé sur le véhicule. Il m'a été possible de contrôler les blocs optiques grâce à un ordinateur ou un téléphone portable.»

Bruno MULLER, filière Informatique et Réseaux. Actuellement en stage de fin d'études (2014).

\section{BARQUETTE 100\% ELECTRIQUE}

Ce prototype est conçu à partir d'une barquette $700 \mathrm{CN}$ biplace (voir Figure 6) construite par la société AILEF. L'objectif de ce projet est de concevoir une chaîne de propulsion électrique. Ce projet est mené actuellement par les membres du club ECARTEAM. Il s'agit d'un projet débuté au cours de l'année scolaire 2012-2013.

\subsection{Caractéristiques techniques}

Le châssis monocoque de la barquette est fabriqué en tôles d'aluminium rivetées avec couples et structure avant en tubes d'acier. Elle comporte les éléments de sécurité passive (arceau de sécurité, protection frontale, harnais 6 points, etc.) en conformité à la réglementation. Le Tableau 3 présente quelques caractéristiques techniques de la barquette d'origine.

\subsection{Chaîne de propulsion électrique}

La propulsion est assurée par un moteur AC-50 Brushless, fonctionnant avec une tension de 72 à $96 \mathrm{~V}$, absorbant un courant nominal de $650 \mathrm{~A}$, fournissant une vitesse de rotation max. de $6000 \mathrm{tr} / \mathrm{min}$ et un couple de 150 N.m. Ce moteur correspond au dimensionnement effectué compte tenu des spécifications souhaitées pour la voiture (vitesse max. de $27 \mathrm{~m} / \mathrm{s}$ ). Ce type de moteur est utilisé pour la propulsion de véhicules électriques de taille moyenne. Il peut être également utilisé comme génératrice lors du freinage permettant de mettre en place la récupération de l'énergie.

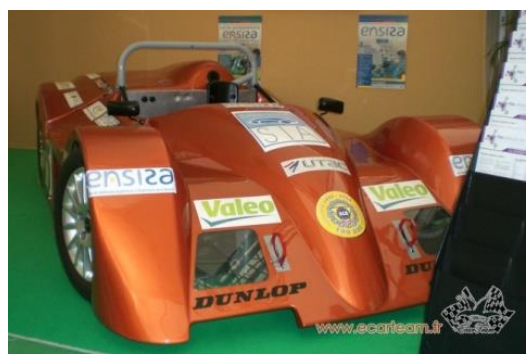

Figure 6 Barquette électrique

\begin{tabular}{|l|l|}
\hline Motorisation & Moteur Renault K4J, 1390 cc \\
\hline Boîte de vitesse & 5 rapports \\
\hline Poids & $520 \mathrm{~kg}$ \\
\hline Long./Larg./H. & $3670 / 1750 / 1000 \mathrm{~mm}$ \\
\hline \multicolumn{2}{|l|}{ Tableau 3. Caractéristiques techniques de la barquette }
\end{tabular}

Afin de pouvoir mettre en place un système de récupération de l'énergie, des batteries lithium-ion ont été choisies. Elles permettent une charge dite rapide comparée à une charge lente des batteries au plomb. Plus précisément, les batteries choisies utilisent la technologie Li-Po (Lithium ion polymère). Par rapport aux batteries Li-ion, les batteries Li-Po sont plus résistantes aux surcharges et aux risques d'explosion. Par ailleurs, elles disposent d'une durée de vie plus importante (nombre plus important de cycles). L'intégration du moteur électrique et des batteries est illustrée sur la Figure 7. Des essais préliminaires en conditions réelles montrent la validité de la solution adoptée ${ }^{4}$.

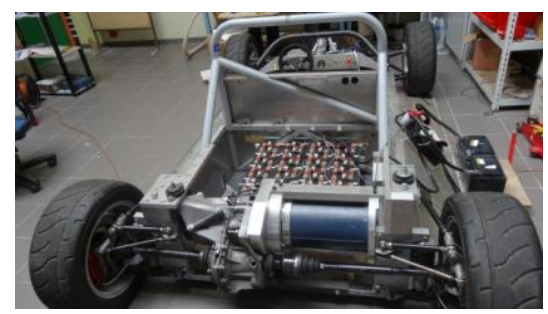

Figure 7 Mise en ouvre de la motorisation électrique

\subsection{Témoignages}

«(...) une expérience très enrichissante, probablement l'expérience que j'ai vécue à l'école la plus proche de ce que je vis aujourd'hui durant mon stage, donc proche du monde professionnel. En effet, la gestion d'une équipe, ainsi que la gestion des fournisseurs,

${ }^{4}$ https://www.facebook.com/photo.php?v=615020988568911\&set=v b. $496709810400030 \&$ type $=2 \&$ theater 
l'anticipation des besoins sans oublier la logistique et la communication (..) avec des hauts et des bas évidemment, mais le tout avec une très forte motivation. (...) Ce genre de projet n'a pu être mené à la légère et a demandé beaucoup de travail (...) aujourd'hui, j'en parle avec un tel enthousiasme et une telle fierté que je ne peux regretter une seconde tout le temps passé. » Aurélien DANEAU, filière Mécanique. Actuellement en stage de fin d'études (2014).

"Pour moi, Ecarteam a été le premier déclic du métier d'ingénieur dès mon arrivée en première année, (...) un partage de connaissances dans un cadre convivial, j'ai découvert le travail en équipe, la gestion d'un projet, l'élaboration d'un planning, la planification des tâches, le démarchage de sponsors, les réunions d'avancement de projet (..) C'est l'avantage que j'ai aujourd'hui dans l'entreprise, par rapport à d'autres stagiaires ; j'ai déjà une expérience du métier d'ingénieur, et je l'ai acquise par moi même. (..) ma participation a été fondamentale dans ma formation d'ingénieur, et inoubliable en tant qu'aventure humaine. »

Marion BORDES MONIER, filière Mécanique. Actuellement en stage de fin d'études (2014).

"Lors de mon arrivée, je me suis intéressé aux clubs étudiants car c'est pour moi une composante importante de la formation d'ingénieur. Passionné d'automobile, EcarTeam a retenu mon attention et j'ai décidé d'y prendre part activement. C'est une expérience très enrichissante. (...) On est également en relation avec les enseignants et techniciens de l'école en dehors du cadre purement scolaire. C'est une belle façon de mettre en pratique ce que l'on nous enseigne mais aussi d'approfondir ses connaissances et de s'ouvrir sur les différentes filières de l'école. (..) parfois chronophage mais toujours gratifiant et cela m'a permis de développer des aptitudes que me seront utiles à l'avenir. » Romain THOLIN, filière Mécanique. Actuellement en $2^{\text {ème }}$ année (2014).

\section{BILAN PEDAGOGIQUE ET DISCUSION}

L'introduction des projets tutorés et la participation aux clubs sont considérées par les étudiants comme des éléments importants dans leur formation d'ingénieur. Il est cependant difficile de quantifier l'impact et la contribution de ces projets sur la formation initiale. On peut néanmoins remarquer que les projets constituent une «carte de visite» lors de la recherche d'un stage ou d'une première expérience dans le monde du travail. Bien souvent, l'expérience acquise lors de la participation à un projet ou un club est très bien perçue par les recruteurs.

Les étudiants qui participent au club sont très motivés car leur participation vient d'une démarche personnelle. Cependant, le déroulement du projet au cours de l'année n'est pas synchronisé avec les enseignements. Il peut y avoir de périodes dans lesquelles le projet avance très peu à cause d'une charge important de travail. Bien souvent, le nombre des membres actifs du club décroît au fur et à mesure que l'année scolaire avance. Les étudiants le plus motivés restent tout au long de l'année et assurent dans la mesure du possible un transfert de connaissances vers les nouveaux membres afin d'assurer la continuité du projet. D'une façon générale, l'utilisation des véhicules comme plateformes expérimentales permet de motiver les étudiants et de les confronter à un problème global d'un projet ingénieur. Des réflexions sur les moyens d'intégrer ces véhicules dans les enseignements classiques de l'école sont actuellement menées.

Nous tirons un bilan global très satisfaisant de cette démarche pédagogique qui nécessite un effort financier relativement important pour l'achat du matériel. Le retour sur l'investissement se fait de plusieurs façons : les étudiants ont une expérience enrichissante, le travail sur ces véhicules est bien apprécié par les industriels, les enseignants ont la possibilité de proposer des projets assez variés, les prototypes peuvent être exposés et constituent une vitrine des réalisations de l'école.

\section{REMERCIEMENTS}

Que toutes les personnes (étudiants, enseignants, personnel technique et administratif, etc.) et en particulier tous les membres du club ECARTEAM qui ont contribué de près ou de loin à l'aboutissement de ces projets soient remerciées.

\section{Bibliographie}

[1] J.-L. Perrin, et al. "Guide du projet tutoré", Guide conçu à l'initiative du Service Universitaire de Pédagogie (SUP) de l'Université Toulouse III. URL : http://sup.upstlse.fr/projettutore

[2] R. Pelletier, R. Lefebvre, "Réussir l'intégration du traitement du signal et des systèmes embarqués par l'intermédiaire d'une approche d'apprentissage par problèmes et par projets", Actes CETSIS 2011, Trois rivières (Québec-Canada), Octobre 2011.

[3] R. Orjuela, et al., "La place des Robots LEGO dans l'enseignement supérieur: un retour d'expérience dans trois contextes différents", Actes CETSIS 2013, Caen (France), Octobre 2013.

[4] D. Matt, et al., "Prototype PGO HEMERA Electrique : Réalisation de la chaîne de traction électrique", Actes CETSIS 2013, Caen (France), Octobre 2013.

[5] R. Orjuela et al., "Réalisation d'un véhicule hybride à partir d'un véhicule grand public", $4^{\text {èmes }}$ Journées Démonstrateurs en Automatique, Angers (France), Juin 2013.

[6] J.-M. Mus, "Le pneumatique dans un environnement «technologie véhicule évolutif»: impacts et perspective", Annales des Mines - Réalités industrielles 3/2009. 